\title{
ANTI-PHASE DRIVEN RATE GYROSCOPE WITH MULTI-DEGREE OF FREEDOM SENSE MODE
}

\author{
Adam R. Schofield ${ }^{1}$, A lexander A. Trusov ${ }^{1}$, C enk A car $^{2}$, and Andrei M. Shkel ${ }^{1}$ \\ ${ }^{1}$ Department of $M$ echanical and A erospace Engineering, University of California, Irvine, \\ Irvine, CA, USA \\ (Tel : +1-949-824-6314; E-mail: aschofie@uci.edu, atrusov@uci.edu, ashkel@uci.edu) \\ ${ }^{2} \mathrm{~B} E \mathrm{I}$ Systron Donner A utomotive Division, Concord, CA, USA \\ (Tel : +1-949-412-5921; E-mail: cacar@ systron.com)
}

\begin{abstract}
Previously, we reported a 3-DOF (degree of freedom) rate gyroscope design with a 1-DOF drive and 2-DOF sense mode that was shown to be robust to environmental fluctuations. In this work, we introduce a novel design concept that combines the robustness of multi-DOF sensing with the common mode rejection of tuning fork devices in the anti-phase driven 6-D OF gyroscope. Prototypes of the design were fabricated and characterized for both rotational and acceleration inputs. For acceleration loads, the device responded in a common mode resulting in a 75\% reduction in amplitude for a differential signal, while for rotations, it responded in anti-phase with sensitivities of $1.687 \mu \mathrm{V} / \mathrm{deg} / \mathrm{s}$ and $-1.887 \mu \mathrm{V} / \mathrm{deg} / \mathrm{s}$.
\end{abstract}

Keywords: inertial sensors, gyroscope, tuning fork, robust design

\section{INTRODUCTION}

In many applications, including automotive and defense, gyroscopes are subject to wide-varying environmental conditions where robustness to parameter fluctuations and ambient vibrations is critical. M any commercially available gyroscopes rely on the inherent ability of tuning fork designs to reject common mode inputs; robustness to environmental fluctuations, however, is generally addressed through control systems [1,2].

Previously, we presented a gyroscope concept that increased the degrees of freedom (DOF) in the sense mode to provide a non-resonant, stable gain region which was shown to be robust to temperature variations [3]. We now propose a 6-DOF robust tuning fork design that takes advantage of two 3-DOF devices coupled in the drive mode.

Prototypes of the 6-DOF gyroscope concept were fabricated using an in-house, wafer-scale SOI process. The anti-phase drive mode dynamics was experimentally characterized and the sensor response was tested for both rotation and acceleration inputs.

\section{DESIG N CONCEPT}

A schematic of the 6-D OF gyroscope concept is shown in Fig. 1. The design consists of two systems, labeled left mass and right mass, which are both 3-DOF gyroscopes connected via a coupling suspension. The coupling forms a 2-DOF system in the drive mode so that the complete device forms a 6-DOF dynamic system.

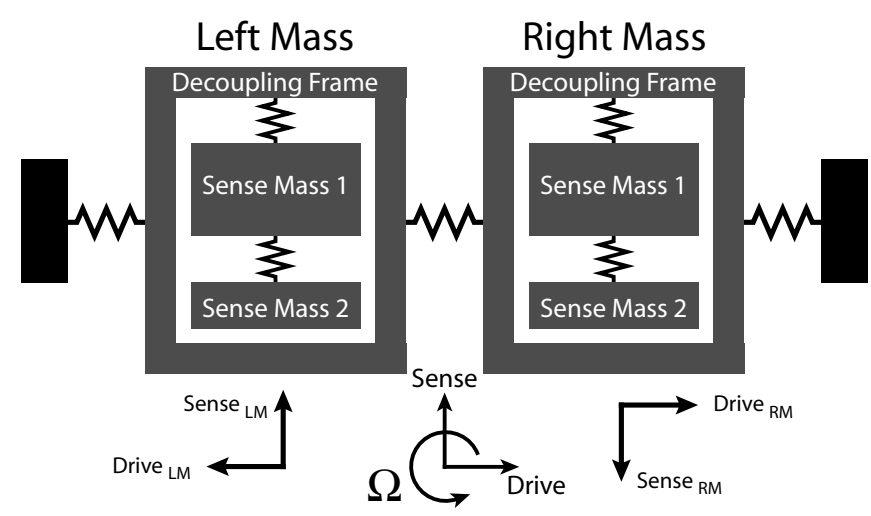

Fig. 1 Schematic of 6-DOF gyroscope concept.

The designed frequency response of the device is shown in Fig. 2. The drive system has two resonant modes: an in-phase peak and a higher frequency anti-phase peak. The device is intended to operate at the anti-phase resonant frequency and the coupling in the drive mode ensures that resonance occurs in both the right and left mass at the same frequency. By driving the masses in antiphase, their corresponding sense signals will also be in anti-phase; this, like other tuning fork designs, is what will allow the 6-DOF device to reject common mode inputs. 


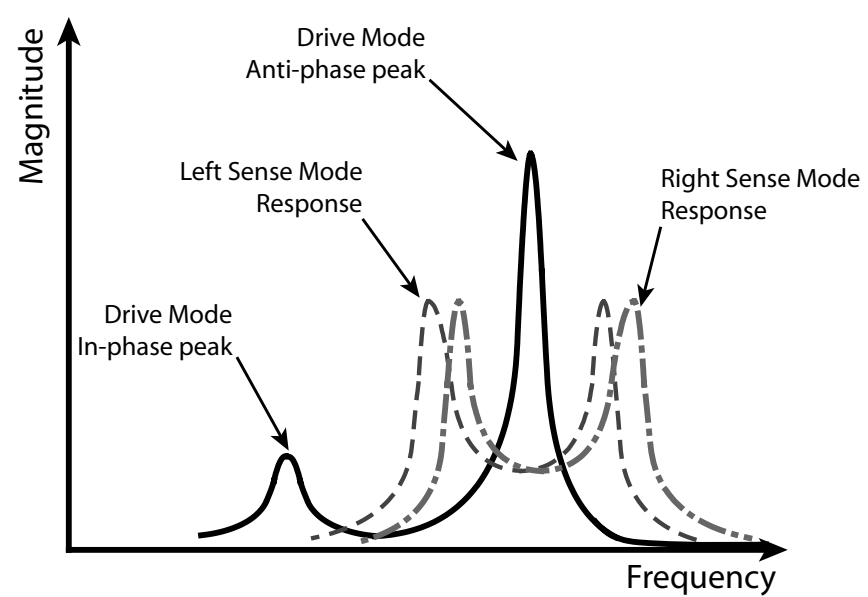

Fig. 2 Conceptual image of the expected magnitude of the drive and sense frequency responses.

The sense modes are also 2-DOF systems where the resonant frequencies are designed to be symmetric about the drive mode anti-phase resonance. This creates a region in the frequency response between the peaks that has both gain and phase stability, which provides the device robustness to variations in operational parameters by design. Thus, the device takes advantage of both tuning fork drive operation as well as the improved robustness of multi-DOF sense systems.

\section{FABRICATION}

Prototypes of the 6-DOF gyroscope concept were fabricated using an in-house wafer level SOI process with a conductive $50 \mu \mathrm{m}$ device layer. AZ photo-resist was spin-coated onto the wafer and patterned using a photo-mask with a Karl Suss MA 6 exposure system. A fter photo-resist development, the wafers were subjected to a timed Deep Reactive Ion Etching (DRIE) step using a Surface Technology Systems (STS) Advanced Silicon Etching (ASE) tool. The minimum feature size of the process was $5 \mu \mathrm{m}$ which was used to define the capacitive gaps. A timed HF etch was then used to release the mobile structures. The device was packaged in a 24-pin ceramic DIP package and wire bonded for the experimental characterization presented below.

Fig. 3 shows an SEM micrograph of a fabricated 6-DOF gyroscope. The design uses mechanical mode decoupling between the drive and sense modes, where the sense masses are suspended relative to a decoupling frame. Each sense system consists of two masses, a larger mass (M ass 1 ) and a smaller mass ( $M$ ass 2 ) with parallel plate capacitors for Coriolis sensing. A common bank of central lateral combs is used to provide the anti-phase drive forcing to the left and right masses simultaneously.

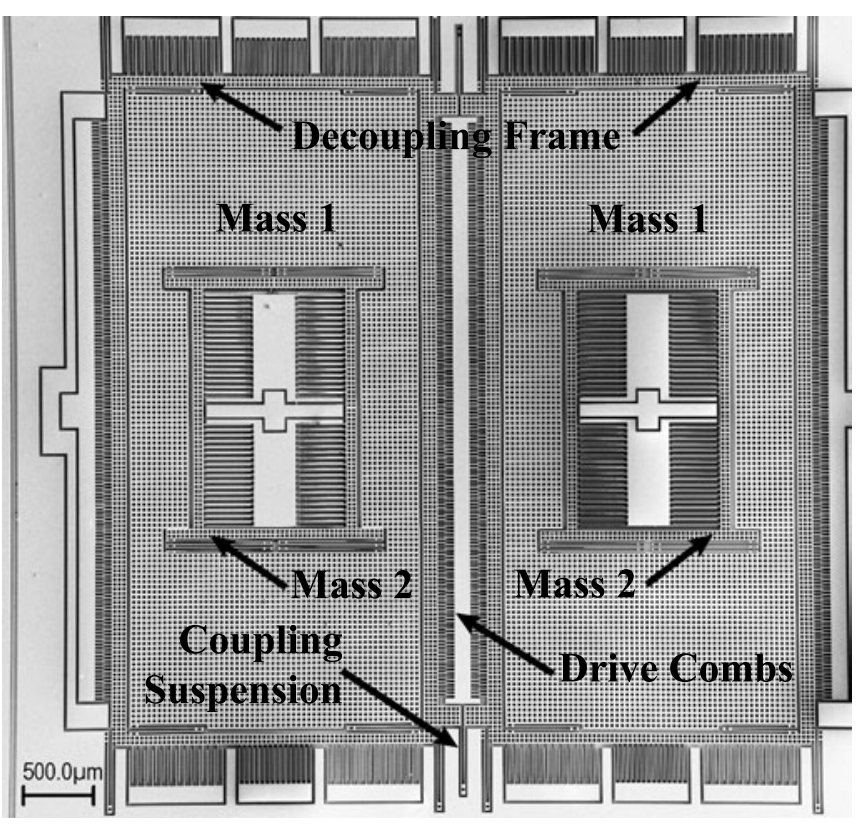

Fig. 3 SEM image of a fabricated 6-DOF gyroscope prototype.

\section{EXPERIMENTAL RESULTS}

A biasing schematic is presented in Fig. 4 that depicts the electrical inputs and outputs of the gyroscope used for the experiments presented below. The inputs to the system are voltages which are defined as the voltage applied the mass, $V_{m}$, and the voltage applied to the central bank of lateral combs, $V_{d}$. The outputs of the system are the motional sense currents across the lateral comb capacitors for the drive mode and the parallel plate capacitors in the sense modes. These currents are amplified and converted to voltages using transimpedance amplifiers, resulting in the left and right drive mode sensing voltages, $V_{D L}$ and $V_{D R}$, and the left and right sense mode sensing voltages, $\mathrm{V}_{\mathrm{SL}}$ and $\mathrm{V}_{\mathrm{SR}}$. 


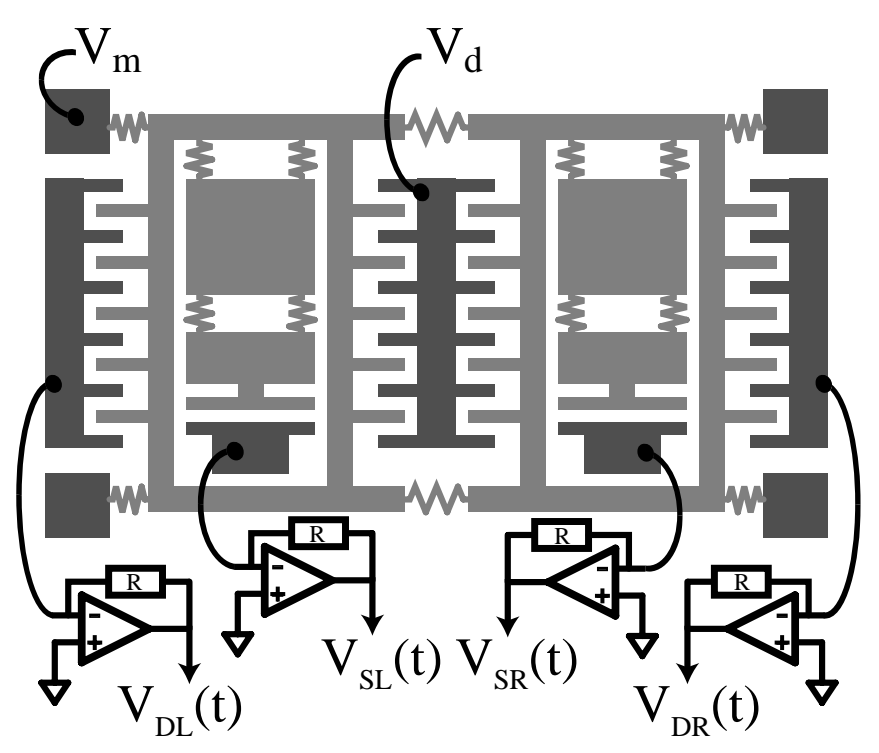

Fig. 4 Biasing schematic used for experimental characterizations of 6-DOF prototypes.

\subsection{Frequency Response}

In order to verify the design concept, the frequency response of the drive mode was experimentally characterized. For this experiment, a parasitic free sweep was employed where electromechanical amplitude modulation (EAM) was used to extract the motional signal from the drive feed-through as detailed in [4]. A $3.535 \mathrm{Vrms} \mathrm{AC}$ driving voltage combined with a $25 \mathrm{~V} D C$ bias was applied to $V_{d}$, while a carrier $A C$ voltage of 3 V rms at $20 \mathrm{kHz}$ that was applied to $\mathrm{V}_{\mathrm{m}}$. The device was actuated at room temperate and atmospheric pressure. The drive mode sensing voltages, $V_{D L}$ and $V_{D R}$, were first demodulated at the carrier frequency and then sent to an HP 35665A Dynamic Signal A nalyzer resulting in the frequency responses presented in Fig. 5.

The magnitude plot reveals two resonant frequencies: a small amplitude peak at $1.44 \mathrm{kHz}$ and a large amplitude peak at $2.167 \mathrm{kHz}$ corresponding to the in-phase and anti-phase modes. The anti-phase mode is characterized by a common phase transition which results from the device layout where the drive mode sense capacitors are oppositely oriented. Due to anti-phase driving, the in-phase resonant amplitude is minimized relative to the anti-phase mode. A Iso, the magnitude of the left mass is larger than that of the right mass. This can be attributed to asymmetries introduced by fabrication imperfections and can be compensated by adjusting the individual amplifier gains.
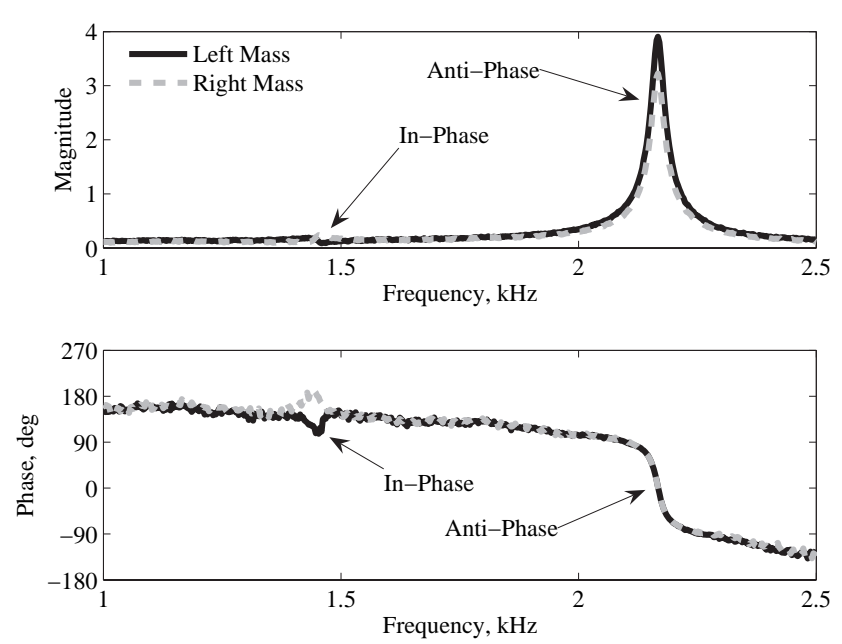

Fig. 5 Experimentally obtained magnitude and phase of the drive mode frequency response.

\subsection{A cceleration Input}

In order to demonstrate the common mode rejection of the 6-DOF concept, the device was subject to an acceleration load. A mechanical impulse was applied and the responses of each sense system were monitored. For this experiment, a constant $D C$ bias of $10 \mathrm{~V}$ was applied to $\mathrm{V}_{m}$ while the sense mode outputs, $V_{S L}$ and $V_{S R}$, were monitored using a LeCroy WaveSurfer Model 452 Digital Oscilloscope.
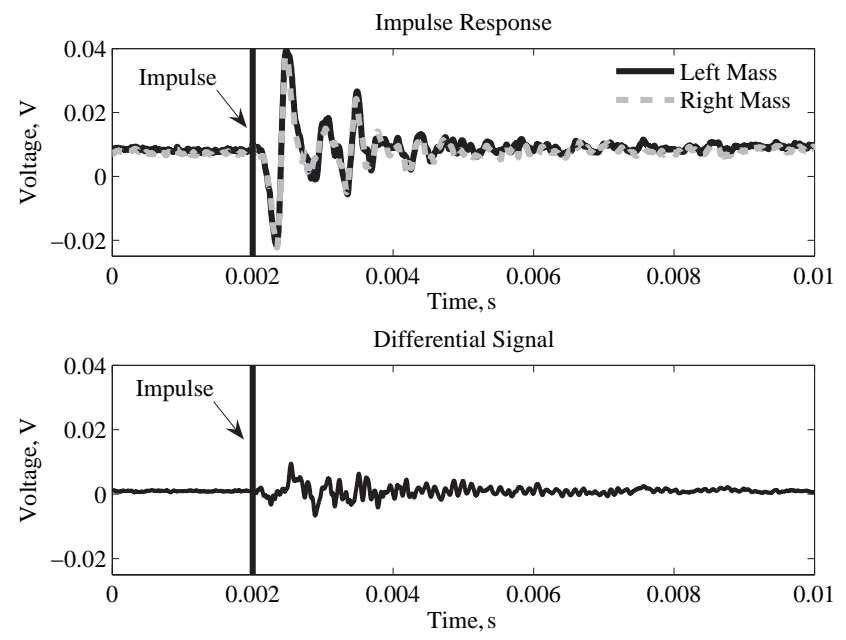

Fig. 6 Experimentally obtained impulse response (top) and the differential signal (bottom).

Fig. 6 (top) shows the time history of the left and right masses due to an applied acceleration impulse. The response of the right mass tracks the left mass as expected. The difference of the two signals is shown in Fig. 6 (bottom) on the same 
scale. The differential results in a $75 \%$ reduction in amplitude versus the individual outputs. The differential signal can be improved further by signal conditioning and calibration.

\subsection{Rotational Input}

The 6-DOF gyroscope prototype was also characterized under constant rotational inputs. For this experiment, a two stage demodulation technique was employed which uses EAM to extract the Coriolis signal from the drive feed-through. Two A dvanced M easurement Technology Model 7265 lock-in amplifiers were used to generate the drive and carrier signals. The voltages used were 3.535 V rms AC at $2.167 \mathrm{kHz}$ combined with a $40 \mathrm{~V} \mathrm{DC}$ bias applied to $\mathrm{V}_{\mathrm{d}}$, while an $\mathrm{AC}$ voltage of $3 \mathrm{Vrms}$ at $18 \mathrm{kHz}$ was applied to $\mathrm{V}_{\mathrm{m}}$. The device was placed on an I deal A erosmith 1291BR rate table at room temperate and atmospheric pressure and tested for constant angular rates of $\pm 300 \mathrm{deg} / \mathrm{s}$.

The sense mode voltages, $\mathrm{V}_{S L}$ and $\mathrm{V}_{S R}$, were demodulated twice, first at the carrier frequency then at the drive frequency to produce the DC output voltages plotted in Fig. 7. A linear fit was performed for both masses resulting in a measured sensitivity of $-1.887 \mu \mathrm{V} / \mathrm{deg} / \mathrm{s}$ and linearity of $0.68 \%$ FSO for the left mass and a measured sensitivity of $1.687 \mu \mathrm{V} / \mathrm{deg} / \mathrm{s}$ and linearity of $1.4 \%$ FSO for the right mass.

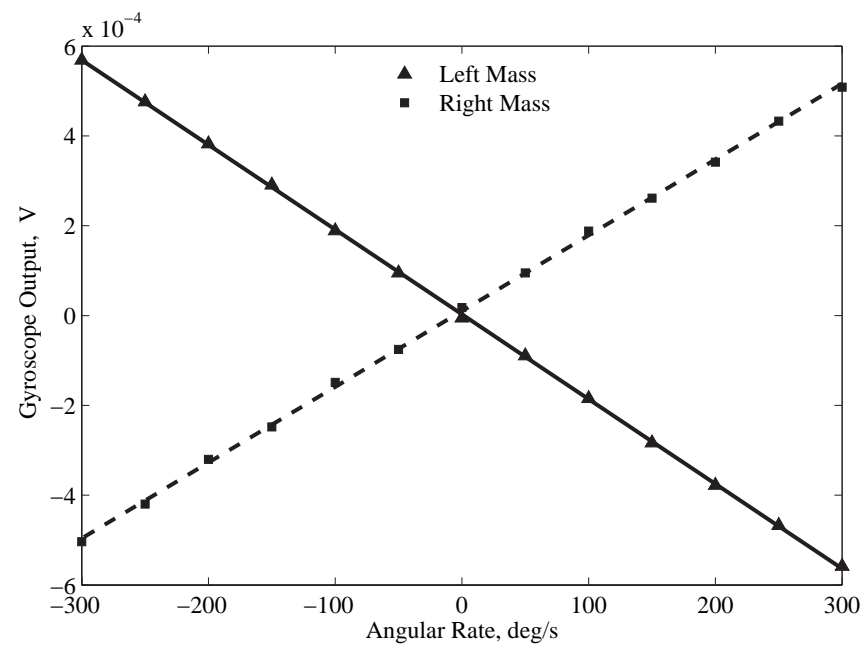

Fig. 7 Constant rate response for right and left masses of 6-DOF gyroscope.

The responses of the left and right masses have sensitivities with opposite signs as expected for the anti-phase driving. The total output of the gy- roscope would be the differential of the two sense signals which would result in an overall increase in the total output. The sensitivity and full scale output of the left mass was larger than the right mass which, as stated above, can be attributed to imperfections of the fabrication process. This can be compensated by adding drive mode amplitude control and ensuring proper calibration of both sense masses.

\section{CONCLUSIONS}

A novel gyroscope concept based on robust multi-DOF sensing and anti-phase driving was introduced. Prototypes of the 6-DOF design were fabricated and characterized. Experimental results were presented which demonstrated the ability of the device to reject common mode inputs while remaining sensitive to Coriolis accel erations.

\section{ACKNOWLEDGEMENTS}

This work was supported by BEI Technologies contract BEI-36974 and UC Discovery program ELE04-10202. The authors would like to thank J ohn Porter of the Carl Zeiss Center of Excellence for use of the UItra 55 SEM and Vu Phan of the $\mathrm{UCI}$ Integrated Nanosystems R esearch Facility.

\section{REFERENCES}

[1] J. A. Geen, "Very low cost gyroscopes," IEEE Sensors 2005, Irvine, CA, USA, 2005, pp. 537540.

[2] R. Neul, U. Gomez, K. Kehr, W. Bauer, J. Classen, C. Doring, E. Esch, S. Gotz, J. Hauer, B. K uhlmann, C. Lang, M. Veith, and R. Willig, "M icromachined gyros for automotive applications," IEEE Sensors 2005, Irvine, CA, USA, 2005, pp. 527-530.

[3] C. A car and A. M. Shkel, "Inherently robust micromachined gyroscopes with 2-DOF sensemode oscillator," J. Microelectromech. Syst., vol. 15, no. 2, pp. 380-387, 2006.

[4] A. Trusov, C. A car, and A. M. Shkel, "Comparative analysis of distributed mass micromachined gyroscopes fabricated in SCS-SOI and EFAB," ProC. of the SPIE: Smart Structures and $M$ aterials 2006, vol. 6174, San Diego, CA, USA, 2006, pp. 61742A. 